\title{
Infrarenal Aortoiliac Aneurysm In Young Female With No Predisposing Factor: A Case Report
}

\author{
Sarvesh Sharma ${ }^{1}$, Anula Sisodia ${ }^{2}$, Sanjeev Devgarha ${ }^{3}$, \\ Rajendra Mohan Mathur ${ }^{4}$ \\ ${ }^{1}$ Resident, Department Of Ctvs, S.M.S Medical College, Jaipur Rajasthan, India \\ ${ }^{2}$ Assistant Professor, Department Of Ctvs, S.M.S Medical College, Jaipur Rajasthan, India. \\ ${ }^{3}$ Associate Professor,Department Of Ctvs, S.M.S Medical College, Jaipur Rajasthan, India. \\ ${ }^{4}$ Professor And Head, Department Of Ctvs, S.M.S Medical College, Jaipur Rajasthan, India.
}

\begin{abstract}
Abdominal aortic aneurysm is a common and potentially fatal condition upon rupture, that warrants screening, early diagnosis and treatment to reduce mortality. There are various risk factors, which causes aortic aneurysm. They are smoking history, male gender, old age, family history of AAA, coronary artery disease, COPD etc.

Here we present a case of 30 years old female having abdominal aortic aneurysm despite not having any risk factors or general causes of abdominal aortic aneurysm. Pt. was operated by transperitoneal approach and aortobiiliac Anastmosis was done with PTFE graft. Post operative period was uneventful.
\end{abstract}

\section{Introduction}

Aneurysms are defined as a focal dilatation at least 50\% larger than the expected normal arterial diameter. Abdominal aorta is a common site of aneurysmal dilatation. A practical working definition of an AAA is transverse diameter $3 \mathrm{c} . \mathrm{m}$. or greater and for a common iliac artery aneurysm a transverse diameter $1.8 \mathrm{c} . \mathrm{m}$. or greater AAAs more accurately are referred to as degenerative or non specific in etiology. Less frequent causes include infection, cystic medial necrosis, arteritis, trauma, inherited connective tissue disorders and pseudo aneurysm from anastmotic disruption. They increase steadily in frequency after age 50, are two to six times more common in men than in women, and two to three times more common in white men than in black ones. Increased risk factors for AAA are smoking History, family history of AAA, older age. Coronary artery disease, high cholesterol, COPD, high (per 7 c.m. interval). Decreased risk factors are deep vein thrombosis, DM, Black race and female gender.

\section{Case Report}

A-30 years old female was admitted with complain of abdominal pain. The patient reported with history of pain in the central part of abdomen for last 6 months. Pain was constant, dull aching in nature. Patient also had backache. Severity of pain increased on exertion. There were no bowel and bladder dysfunctions. Patient had no history of vomiting, diarrhea. Patient had no history of previous long term hospitalization, no history of previous surgery. Patient had no history of DM, Hypertension. COPD, T.B., epilepsy and any other chronic diseases. There was no family history of such kind of disease. Patient had three children. Patient was nonsmoker, vegetarian and non alcoholic.

On physcial examination, there was a pulsatile swelling present in ublical region of 5 c.m. x 5 c.m. size. Temprature of skin over swelling was normal, tenderness present. The swelling had expensile pulsation. Temperature of both lower limbs was lower than rest of body temperature. All peripheral pulses were palpable. Her heart rate was 80 beats/min. and blood pressure was 126/78 mm. of Hg. Her ESR was with in normal limits.

USG of whole abdomen showing dilatation of terminal end of aorta $4.5 \mathrm{c} . \mathrm{m}$. length, measurement of aorta at dilatation was $26 \mathrm{~mm}$., calcified plagues seen in intima. CT chest and abdominal angiography showed aneurysmal dilatation of lower most part of abdominal aorta and involving the origin of bilateral common iliac arteries with calcified plagues in the wall of the dilated segment. $2 \mathrm{D}$ echo of patient showed normal study.

After all routine investigations, patient was operated by trans peritoneal approach. Abdominal aortic aneurysm of size $5 \mathrm{c}$.m. x 7c.m. was found at lower part of aorta extending upto origin of common iliac arteries bilaterally. After incising the aneurysm abdominal aorta and both iliac arteries were anastmosised with PTEE graft. Post operative period was uneventful. Both lower limb had same temperature as other parts of body at the time of discharge. 
Fig. 1: showing angiographic film of abdominal aortic aneurysm.

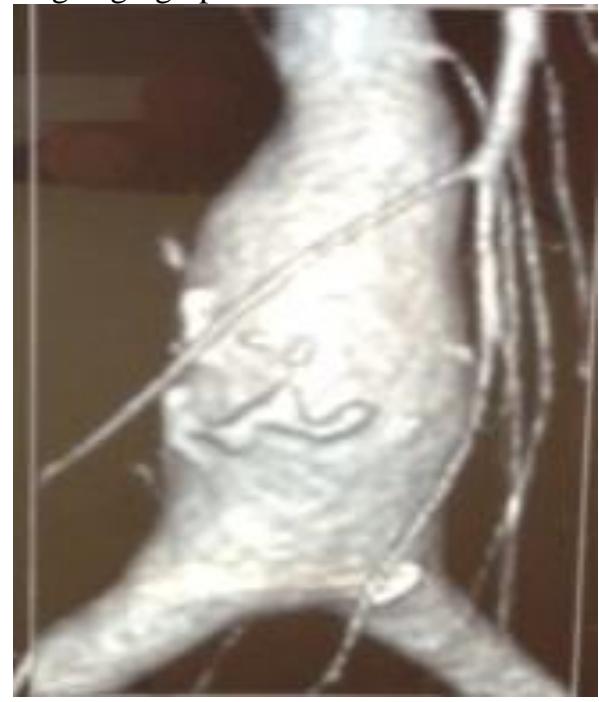

\section{Discussion}

AAA is relatively common and with repture it is often fatal. The primary pathogenesis of an AAA involves degradation of extracellular matrix, protein, elastin and collagen, resulting in the remodeling of the aortic wall ${ }^{1}$. Elastin is the principal load bearing relment in the aorta that resists aneurysm formation, elastin in not synthesized in the adult aorta but has a half life of 40-70 years, accounting for its reduction with age and the occurance of AAA's primarily in elederly patients ${ }^{1}$. Degenerative aneurysms account for more than $90 \%$ of all infrarenal AAAs. Less frequent causes include infection (Syphilitic and mycotic) cystic medial necrosis, arteritis, trauma, inherited connective tissue disorders, marfan's syndrome, auto immune disease and haemodynamic mechinical factors have been implicated in the pathogenesis of AAAs ${ }^{2,3}$.

The pathogenesis of AAA has been frequently associated with atherosclerosis ${ }^{2,4}$. Athesosclerosis is a form of arteriosclerosis in which atheromas containing cholesterol, lipoid material, and lipophages are formed with in the intima and inner media of a large and medium sized artery. Hypertension in considered to be a predisposing factor to AAA. ${ }^{5}$

Independent increase risk factors for detecting obdominal aortic aneurysm on ultrasound screening are smoking story, family history of AAA, older age, Coronory artery disease, male gender high cholesterol, COPD, hight (Per $7 \mathrm{~cm}$. interval). Decreased risk factors are abdominal imaging with in 5 years, Deepvein thrombosis, Diabetes mallitus, Black race, female gender. ${ }^{1}$

Current treatment guidelines recommend repair of AAA when aneurysm reacher $5.5 \mathrm{~cm}$. in maximum diameter or expand by more than $0.6-0.8 \mathrm{~cm}$./year ${ }^{6}$ The most common site of AAA is at the bifurcation of aorta into the common iliac arteries. ${ }^{2}$ Male are reported four times frequent than female. ${ }^{4}$ The median age of rupture was 76 in men and 81 in woman. ${ }^{1}$ The median AAA size at rupture was $8 \mathrm{~cm}$. but $4.5 \%$ of the ruptured AAA were less than $5 \mathrm{~cm}$. in diameter.

\section{References}

[1] R.B. rutherford : Vasculare surgery ( $6^{\text {th }}$ edition $)(1409-1412)$.

[2] Vernon LF, peacock JR, Esposito AP: Abdominal aortic aneurysms presenting as low back pain: a report of two cases. JMPT 1986; 9(1) : 47-50.

[3] Desforges JF: Abdominal aortic aneurysms. New England J.med 1993; 328 (16): 1167-1172

[4] Stites J, Canterbury R.: Aneurysm of the abdominal aorta. Aca J. chiropractic. Nov. 1989; 65-67.

[5] Boline PD, Andesson AV: Abdominal aortic aneurysm. AJCM 1989: 2(3) 114-116.

[6] Brewster DC, Cornenwett JL : Hallett JW Jr. etc. al; Guideline for the treatment of abdominal aortic aneurysms. Report of a subcommitttee of the joint council of the American association for the vascular surgery and soicety for vascular surger. $\mathrm{J}$ vase surg. $2003: 37(5): 1106-1117$

[7] Abdominal aortic aneurysm: Case report camille Hadida, DC, FCCS(C) moez Rajwani, Bsc. DC: (JCCA 1998; 42(4) 216-221)

[8] AVDLA Vithanage, MCB Galanitiyawa, JDVC Lekamwasam, sarath Lekamwasam: Inflammatory abdominal aortic aneurysm; a case report (Galle medical journal Vol. 13; No. 1, Sept. 2008) 